\title{
Proposal for the 4th Workshop on Mobile Gaming, Mobile Life - Interweaving the Virtual and the Real
}

\author{
Barbara Grüter $^{1}$, Holger Mügge ${ }^{2}$, Leif Oppermann ${ }^{3}$, and Mark Billinghurst ${ }^{4}$ \\ ${ }^{1}$ Hochschule Bremen, Flughafenallee 10, 28199 Bremen, Germany \\ barbara.grueter@hs-bremen. de \\ ${ }^{2}$ Universität Bonn, Römerstraße 164, 53177 Bonn, Germany \\ mueggeaiai.uni-bonn. de \\ ${ }^{3}$ Fraunhofer FIT, Schloß Birlinghoven, 53754 Sankt Augustin, Germany \\ leif.oppermannafit.fraunhofer.de \\ ${ }^{4}$ HIT Lab NZ, University of Canterbury, Private Bag 4800, Christchurch 8140, New Zealand \\ mark.billinghurst@hitlabnz . org
}

\begin{abstract}
Over the last few years we have witnessed the smartphone dominating the market, the rapid growth of mobile apps, a surge in mobile augmented reality and location-based apps, and burgeoning mobile communities. While mobile topics continue to provide rich research challenges, people and companies outside academia already use these apps regularly. This is due to the increased availability of affordable devices, applications and technologies that support the creation of mixed reality experiences. Thus a core theme of our workshop at ICEC 2012 is authoring mixed realities, designing mobile games and creating mobile experiences. We would like to assess how professional designers and developers, as well as academics and end users, are using the technology to connect the digital and the real in a mobile context.
\end{abstract}

\section{Introduction}

This is the fourth workshop on Mobile Gaming since 2008. Recent developments in the mobile domain emphasize the vanishing border between mobile gaming and mobile life, and the emerging mixed reality worlds, so this fourth installment of the series introduces a revised title and a new member to the committee (Mark Billinghurst). The organizers are from four different organizations and have been actively involved in the field of mobile gaming for at least more than five years each.

\subsection{Organizers Background}

Barbara Grüter is Professor for Human-Computer Interaction (HCI) at the Hochschule Bremen and Associated Professor of the Graduate School Advances in Digital Media at the University Bremen. She is head of the research group Gangs of Bremen with the focus on interaction and mobile gaming experiences by exploring, developing, and play testing mobile game prototypes. She currently leads the BMBF project 
Landmarks of Mobile Entertainment (2010-2013) and is spokeswoman of the research cluster Mobile Life at the Hochschule Bremen since February 2012.

Holger Mügge is a project manager at the University of Bonn and organizes the research group Software Architecture and Middleware which does research projects on context-sensitivity and mobile gaming. He is also co-founder and CEO of Qeevee, a young company that specializes in mobile distributed applications for entertainment, gaming and learning.

Leif Oppermann is deputy head of the Mixed and Augmented Reality Solutions group at Fraunhofer FIT. Through his current role at FIT and previously at the University of Nottingham's Mixed Reality Lab, he has been directly involved in various projects that researched into Mixed Reality and Pervasive Gaming.

Mark Billinghurst is director of the HIT Lab New Zealand at the University of Canterbury. He has a wealth of knowledge and expertise in human computer interface technology, particularly in the area of Augmented Reality and mobile AR. His research team developed the first collaborative AR application for mobile phones, and one of the first mobile AR advertising campaigns.

Together, the organizers have experience in organizing three previous editions of this workshop; two of them as part of the annual conference of the German "Gesellschaft für Informatik" (GI), and one at ACM ACE in 2011.

\subsection{Goals}

This workshop provides a forum for researchers and practitioners in the field of pervasive, mobile, and location-based gaming. The thematic focus is on mobile games and other social mobile applications that take location and context, and players' movements as important design parameters. The workshop particularly addresses mobile game designers, developers, and scholars who are concerned with current and future issues of mobile game design, development, staging, and evaluation. It aims to span the boundaries between play, research, and business.

\subsection{Motivation}

Mobile applications already pervade our everyday life. Mobile games allow their players to exploit the arising opportunities for mobile human-computer interaction in a playful fashion. The archetypical mobile game is Geocaching, which started immediately after the US government announced the discontinuation of the artificial degradation of GPS signals for non-military users on 1 May 2000. In this game, players hide caches, small treasures, and announce their locations as GPS coordinates over the Internet for other players to search for them. In a similar way, mobile games today utilize the location by means of mobile and pervasive technology. Small and portable devices are equipped with sensors and models of their environment that allow them to sense the emerging context of play and interact with the player.

These mobile games combine the real and virtual worlds. As outdoor games they continue the tradition of rallies and Geocaching. They use the outside real environment as playground but use computing devices to access a digital world and to enable 
and organize play activities. Thus they offer unique gaming possibilities. They enable communication and collaboration between players independent from their positions; they augment the real world by game-specific information and action possibilities; they allow the collection of game process data for analysis and complex feedback; they integrate the game interaction with other web-based services and perform complex game mechanics either on the client- and/or the server-side.

In this workshop, we explore the mobile games of tomorrow and their aesthetical, technological and conceptual assumptions. We have already seen the convergence of mobile, social, and map technologies. We are now witnessing the emergence of mobile game communities, the localization of social networks, a surge of mobile Augmented Reality (AR), social mobile gaming platforms, mobile game creators, and the development of dynamic map technologies. We further notice emerging ecosystems connecting mobile gamers, producers, researchers, and game technologies.

\section{The Workshop}

We solicit research or position papers, prototype demonstrations, and position statements and questions as contributions to the workshop. We will set up a program committee and organize at least three reviews for each contribution. Accepted papers and demonstrations will be presented at the workshop as part of the program. In addition we will invite one or two keynote speakers from academia and industry.

During the hands-on session, participants could also try out specific approaches to planning and creating their own mobile experiences under the supervision of an expert.

All contributions will be available to all participants in advance through a workshop webpage which will also be updated after the workshop to reflect the results.

We are expecting about $10-20$ participants. We are aiming for attracting submissions from fitting research projects and student works. Moreover we intend to invite industry representatives, including also small companies.

\subsection{Topics of Interest}

Topics of interest include, but are not limited to:

- $\quad$ Authoring solutions and game creators

- Mobile game design and patterns of game play

- Mobile Augmented Reality/Mixed Reality

- Mobile play experience, design and evaluation

- $\quad$ Producing mobile games

- Interfaces and interaction techniques for mobile games

- Development processes and architectures

- Complexity of hardware, software, infrastructure - problems and solutions

- Mobile games and mobile projectors

- Casualty of mobile games

- Mobile Gamification, Serious Games and Games with a purpose 
- The emerging context of mobile game play and context-sensitive technology

- Market strategies and business models for mobile games

- Staging and deployment strategies

\subsection{Submission, Dates and Websites}

All workshop submissions will be handled via our EasyChair account, which is linked in from the workshop website.

Submission Deadline

Notification of acceptance

Final version

Workshop

Workshop website

Conference website

$\begin{array}{lll}\text { June } & 16 & 2012 \\ \text { July } & 01 & 2012 \\ \text { July } & 15 & 2012 \\ \text { September } & 26-29 & 2012\end{array}$

http://sam.iai.uni-bonn.de/moga2012/ http://www.icec2012.org/

Presented papers will be archived at the workshop website and selected papers will be published with Springer. 